\title{
Efectividad de la participación del profesional de enfermería en los centros de enseñanza especial ${ }^{1}$
}

Institución: Universidad de Costa Rica, Maestría en Enfermería Pediátrica

Dayanna Arias Fonseca ${ }^{2}$

\begin{abstract}
RESUMEN
Enfermería en el ámbito escolar es un área relativamente nueva en Costa Rica. Las autoridades en el país han elaborado una legislación y planteado estrategias en materia de salud, niñez y derechos que destacan la relevancia de intervenciones que favorezcan la salud, educación y desarrollo de la niñez y la adolescencia con discapacidad, por lo que la atención de enfermería en el ámbito de educación especial involucra tanto las intervenciones con esa población así como las investigaciones. El objetivo de este trabajo es analizar la mejor evidencia científica disponible respecto de la efectividad de la participación educativa y cuidado físico por parte de enfermería al estudiantado en centros de enseñanza especial para prevenir complicaciones de salud y potenciar el desarrollo de los infantes con algún tipo de discapacidad: se trata de un protocolo de revisión breve, el cual utiliza la metodología de práctica clínica basada en la evidencia, que aplica los cinco pasos que son: 1. Planteamiento de la pregunta clínica 2. Búsqueda en bases de datos 3. Análisis crítico de la evidencia 4. Transferencia de la evidencia 5. Evaluación.
\end{abstract}

Palabras claves: discapacidad, enfermería, educación-especial

\footnotetext{
${ }^{1}$ Fecha de recibido: 5 de diciembre del 2017

Fecha de aceptación: 16 de marzo del 2018

${ }^{2}$ Enfermera, estudiante de Maestría en Enfermería Pediátrica con énfasis en niñez y adolescencia. Centro Nacional de Educación Especial Fernando Centeno Güell. Universidad de Costa Rica, Costa Rica. Correo electrónico: day1909@hotmail.com
} 


\title{
Effectiveness participation of the nursing professional at the special education centers ${ }^{1}$
}

Institution: University of Costa Rica, Postgraduate Pediatric Nursing

Dayanna Arias Fonseca ${ }^{2}$

\begin{abstract}
Nursing in the school setting is a relatively new area in Costa Rica. The authorities in the country have drafted legislation and proposed strategies in health, childhood and rights that highlight the relevance of interventions that favor the health, education and development of children and adolescents with disabilities. This is how nursing care in the field of special education plays an important role in this population as well as in research. The objective is to analyze the best scientific evidence available regarding the effectiveness of educational participation and physical care by nursing students in special education centers to prevent health complications and promote the development of children with disabilities. It is a brief review protocol, using the methodology of clinical practice based on evidence, applying the five steps that are: 1. Approach of the clinical question 2. Search in databases 3. Critical analysis of the evidence 4. Transfer of evidence 5. Evaluation.
\end{abstract}

Keywords: discapacity, nursing, special-education

\footnotetext{
${ }^{1}$ Date of receipt: December 5, 2017

Date of acceptance: March 16, 2018

${ }^{2}$ Nurse, Master's student in Pediatric Nursing with an emphasis in Children and Adolescence. University of Costa Rica, Costa Rica.

E-mail: day1909@hotmail.com
} 


\section{Eficácia da participação do profissional de enfermagem nos centros de ensino especial ${ }^{1}$}

Instituição: Universidade da Costa Rica, Enfermagem pediátrica de pós-graduação

Dayanna Arias Fonseca ${ }^{2}$

\section{RESUMO}

Enfermagem na escola é uma área relativamente nova na Costa Rica. As autoridades do país elaboraram legislação e propuseram estratégias de saúde, infância e direitos que destacam a relevância de intervenções favoráveis à saúde, educação e desenvolvimento de crianças e adolescentes com deficiência. É assim que os cuidados de enfermagem no campo da educação especial desempenham um papel importante nesta população, bem como na pesquisa. O objetivo é analisar as melhores evidências científicas disponíveis sobre a efetividade da participação educacional e do atendimento físico por estudantes de enfermagem em centros de educação especial para prevenir complicações de saúde e promover o desenvolvimento de crianças com deficiência. É um protocolo de revisão breve, utilizando a metodologia da prática clínica baseada em evidências, aplicando as cinco etapas que são: 1. Abordagem da questão clínica 2. Pesquisa em bases de dados 3. Análise crítica da evidência 4. Transferência de evidências 5. Avaliação.

Palavras-chave: discapacidad, enfermagen, educação -especial

\footnotetext{
${ }^{1}$ Data de recepção: 5 de dezembro de 2017

Data de aceitação: 16 de março de 2018

${ }^{2}$ Enfermeira. Estudante de Mestrado em Enfermagem Pediátrica com ênfase em crianza y garota. Universidade da Costa Rica, Costa Rica. Correio eletrônico: day1909@hotmail.com
} 


\section{Revista Electrónica Enfermeria Actual en costa Rica}

\section{INTRODUCCIÓN}

La enfermería en el ámbito escolar es un área emergente a pesar de no ser nueva en su ejercicio tanto en el país $^{2}$-se remonta a 1941- como internacionalmente se ubica en 1902, en New York-. Costa Rica; sin embargo, cada vez más refleja la necesidad de apuntar a un cambio de visión de la salud, máxime considerando el sustento en el marco legal de la convención de los derechos de las personas con discapacidad ${ }^{3}$ y las estrategias planteadas por entes internacionales en materia de salud, niñez y derechos.

De acuerdo con Campos-Vargas², quien en el 2015 planteó un estudio en Costa Rica en el que desarrolló el cuándo, cómo y porqué surge la educación especial en el país, así como cuáles actores participaron de ese proceso y quiénes se beneficiaron, a partir de los registros históricos de la creación de la primera escuela de enseñanza especial del país en 1940, y de la creación de la Dirección Técnica de Enseñanza Especial-, dentro del personal que integraba dicho centro se menciona un director, un médico psiquiatra del Departamento de Higiene Mental, una enfermera y cuatro profesoras, de lo que se afirma que el desarrollo de la educación especial en el país se da por la asociación entre educación y salud.

Respecto del desarrollo de la temática de intervención de enfermería en los centros de educación especial, Arce-Elizondo, Barrientos-Monge, Castillo-Ramírez y Varela-Vindas ${ }^{4}$, en el 2006, plantearon la intervención de la enfermera pediátrica en la Escuela de Rehabilitación Nacional de agosto a noviembre de ese año, de lo que se concluye la relevancia del rol profesional de la enfermera en esta área.

En cuanto a la población de estudiantes que asisten a los centros de educación nacional así como sus características, CONARE $^{5}$ (201, en el Quinto Informe del Estado de la Educación en Costa Rica, describe el panorama de la educación especial en el país y sus características con el objetivo de comprender la capacidad de respuesta de los centros de educación especial en lo referente a la demanda de sus servicios en todo el país, al tiempo que contextualiza la población en la atención educativa.

En torno al tema, es importante destacar que en la actualidad, y desde la fecha en la que se desarrolló este perfil profesional en el primer centro de educación especial ${ }^{2}$, existe la figura de la enfermera en dicho centro educativo quien debe ejercer funciones en el área de la educación pública (lo cual tan solo se cumple en uno de los 23 centros de educación especial en el país), quien es contratada por el Ministerio de Educación Pública.

El desarrollo investigativo sobre el rol de la enfermera en el ámbito escolar es mucho más amplio, como lo evidenciaron Katz y Hayout ${ }^{7}$ (Israel, 2013), quienes desarrollaron una investigación sobre la vinculación de la discapacidad con patologías asociadas y la relevancia e impacto de un programa educativo de enfermería para niños con discapacidades del desarrollo, que responda a las necesidades de esta población.

En el 2014, Litt et al $^{9}$ trabajaron con el efecto del programa de salud y desarrollo infantil en el uso de educación especial en edad escolar en Estados Unidos ofrecida de forma temprana y concluyeron que existe un alto número de demandas insatisfechas para la atención, ya que no se cuenta con los servicios de apoyo para niños con discapacidades que respondan a las necesidades particulares de la población. 


\section{Revista Electrónica Enfermeria Actual en costa Rica}

\section{www.revenf.ucr.ac.cr}

Por su parte, Del Val-Ortega ${ }^{1}$ en el año 2015, en su estudio efectuado en España, cuyo objeto fue definir la salud escolar y su relación con los profesionales de enfermería, plasmó el desarrollo histórico, estado actual e intervenciones desde en un enfoque internacional y se concluye que esta es un área poco estudiada, en vías de crecimiento como disciplina; sin embargo, las intervenciones registradas evidencian numerosos resultados positivos en la prevención de enfermedades y mejoramiento de la salud de la población que recibe estos servicios.

En ese mismo año, en Estados Unidos, Macintosh y Thomas ${ }^{8}$ plantearon la relevancia del desarrollo de programas de prevención, y el abordaje de las necesidades de salud de los niños con necesidades especiales, dados los múltiples desafíos a los que se enfrentan y que requieren la colaboración entre enfermeras, escolares, psicólogos escolares y maestros de educación especial con el fin de ofrecer procesos de aprendizaje eficaces de los niños: los autores brindan recomendaciones para que, de la vinculación del profesional de enfermería con los docentes, surjan coordinaciones en función de la salud mental de los estudiantes.

Luego, en el año 2016, Campos ${ }^{6}$ plantea la relevancia de las intervenciones de los profesionales en enfermería en la promoción de escuelas saludables en España, con el fin de justificar el papel de la enfermera en dicha área: al respecto, concluye que la atención de dicho profesional en los centros educativos es esencial, debido a que la intervención que ofrece es integral, integrada, continua.

Los anteriores referentes ofrecen un panorama del desarrollo de la enfermería en su trabajo con la población de niños y adolescentes en los centros de educación especial.

El planteamiento de la mejor evidencia respecto de la efectividad de la intervención del profesional de enfermería en los centros de enseñanza especial, para prevenir complicaciones de salud y potenciar el neurodesarrollo, se establece desde la búsqueda de evidencia que determine el papel que desempeña el profesional de enfermería en los centros educativos, en favor de la población beneficiaria de este servicio.

En cuanto a la enfermería escolar, es un área poco desarrollada en Costa Rica, ya que en el Ministerio de Educación Pública solo se cuenta con el rol del profesional de enfermería en un centro educativo público a nivel nacional: -el Centro Nacional de Educación Especial Fernando Centeno Güell, uno de los 23 centros de enseñanza especial que atienden a personas con diferentes condiciones de discapacidad, registrados en el Quinto Informe del Estado de la Educación en Costa Rica 5 .

Considerando la cantidad y las características de la población que reciben estos centros educativos en el país, así como las recomendaciones que la UNICEF plantea en el Estado Mundial de la Infancia (2013): Niños y Niñas con discapacidad, respecto de infantes con discapacidad, que es fundamental "ratificar -y poner en marcha la convención sobre los derechos de las personas con discapacidad y la Convención sobre los derechos del niño", con el fin de potencializar la atención que reciben en función de sus derechos y su óptimo desarrollo . De igual manera, el documento aboga por "Luchar contra la discriminación y mejorar la concientización sobre la discapacidad entre el público en general, los encargados de tomar decisiones y las personas que proporcionen servicios esenciales para los niños y los adolescentes en sectores como la salud, la educación y la protección.

Coordinar servicios en todos los sectores a fin de abordar la amplia gama de problemas que enfrentan la niñez y adolescentes con discapacidad y sus familias. 


\section{Revista Electrónica Enfermeria Actual en costa Rica}

Promover un programa de investigación concertado a escala mundial sobre la discapacidad para generar los datos fiables y comparables que son necesarios para orientar la planificación y la asignación de recursos, y para situar más claramente a los infantes con discapacidad en el temario del desarrollo"3.

Los anteriores son cuatro de los planteamientos que ofrecen estrategias para potencializar las habilidades de la población con discapacidad y que son vinculantes respecto del ejercicio de la enfermería, a las que se puede acceder desde intervenciones planificadas, orientadas a las necesidades de la población.

Aunado a estas estrategias, diseñadas con el fin de ofrecer mejores oportunidades a la niñez con discapacidad, debe considerarse lo sensible del desarrollo, así como las implicaciones de un abordaje temprano, como se destaca en El desarrollo del niño en la primera infancia y la discapacidad ${ }^{10}$, documento en el que se afirma que

“para todos los niños, la primera infancia es una importante ventana de oportunidad para preparar las bases para el aprendizaje y la participación permanentes, previniendo posibles retrasos del desarrollo y discapacidades. Y para los niños con discapacidad, es una etapa vital para garantizar el acceso a intervenciones que puedan ayudarlos a desarrollar todo su potencial"'.

Las acciones contempladas en la Convención Sobre los derechos de las personas con discapacidad, de la cual los estados que forman parte cuentan con el compromiso de cumplimiento. Tal es el caso del artículo 24 respecto de la educación en donde se detalla para la población con discapacidad respecto a este derecho ${ }^{3}$ lo siguiente: “c) Se hagan ajustes razonables en función de las necesidades individuales".

Por otro lado, tomando en consideración que los infantes que presentan discapacidad en muchas ocasiones cuentan con patologías crónicas asociadas, parte de sus necesidades individuales pueden referirse a contar con la asistencia de sus necesidades durante su jornada lectiva, como se establece en el inciso e) de este apartado, el cual considera $^{3}$ que "se faciliten medidas de apoyo personalizadas y efectivas en entornos que fomenten al máximo el desarrollo académico y social, de conformidad con el objetivo de la plena inclusión". Aunado a lo anterior, y en lo referente a lo establecido por la Convención de los Derechos de las Personas con Discapacidad, en el artículo 25 Salud, parte b), los Estados parte " ${ }^{3}$ "proporcionarán los servicios de salud que necesiten las personas con discapacidad específicamente como consecuencia de su discapacidad, incluidas la pronta detección e intervención, cuando proceda, y servicios destinados a prevenir y reducir al máximo la aparición de nuevas discapacidades, incluidos los niños y las niñas y las personas mayores", máxime si estos niños y adolescentes requieren de estos apoyos para acceder a la educación pública, gratuita, obligatoria, para permanecer en las aulas y ofrecer el cumplimiento de estos derechos a través de la posibilidad de acceso.

Otro aspecto por resaltar es que -desde el entorno escolar- la prevención cuenta con gran relevancia ${ }^{11}$, ya que "los efectos de la discapacidad afectan a varios sectores, y exigen coordinar los servicios para tener en cuenta toda la amplia gama de problemas que afrontan los niños con discapacidad y sus familias. Un programa coordinado de intervención temprana, en los sectores de salud, educación y bienestar ayudaría a promover la detección temprana y la gestión de las discapacidades. Se ha demostrado que las intervenciones tempranas permiten obtener mayores avances en la capacidad funcional, y al eliminar los obstáculos al comienzo de la vida se disminuye la gravedad del efecto de los múltiples obstáculos a los que hacen frente a los niños con discapacidad". 
A raíz de planteamientos como el anterior, se subraya la relevancia de la participación del profesional de enfermería en los centros de educación especial, como un elemento a favor de esta población, tanto por el perfil que desempeña y las ventajas de contar con el perfil de la enfermera en un centro de educación especial, de modo que se evite complicaciones de salud y se potencie el neurodesarrollo, sin dejar de lado el aporte de implicaciones de la metodología de investigación basada en la evidencia, es decir, la transmisión de conocimiento ", la cual "es considerada un proceso básico y sistemático. Este proceso incluye la publicación o divulgación de las mejores recomendaciones para disminuir la variabilidad clínica e incertidumbre de los profesionales, como las contenidas en las guías de práctica clínica (GPC), las vías clínicas y los protocolos basados en la evidencia. Y también, comprende la identificación de aquellos elementos o condicionantes que pueden facilitar o dificultar su implementación, para modificar aquellas prácticas consideradas inseguras o adversas para el paciente y/o la calidad asistencial"'.

Por otra parte, la divulgación de la eficacia de las intervenciones del profesional de enfermería pediátrica en cuanto a la prevención de complicaciones y potenciación del neurodesarrollo en los estudiantes es fundamental para la población que recibe este servicio educativo, así como para la enfermería pediátrica, debido a que esta área poco desarrollada de la enfermería pediátrica en el país se puede favorecer a raíz de evidenciar su relevancia. Lo anterior queda plasmado en el Estado Mundial de la Infancia 2013: niñas y niños con discapacidad ${ }^{11}$, el cual enfatiza que "un programa coordinado de intervención temprana en los sectores de salud, educación y bienestar ayudaría a promover la detección temprana y la gestión de las discapacidades. Se ha demostrado que las intervenciones tempranas permiten obtener mayores avances en la capacidad funcional, y al eliminar los obstáculos al comienzo de la vida se disminuye la gravedad del efecto de los múltiples obstáculos a los que hacen frente los niños con discapacidad".

Por lo anterior, se ha propuesto el objetivo de analizar la mejor evidencia científica disponible respecto de la efectividad en la participación educativa y cuidado físico por parte de la enfermería que atiende al estudiantado en centros de enseñanza especial para prevenir complicaciones de salud y potenciar el desarrollo.

\section{MÉTODO}

Para el desarrollo de este protocolo se tomó en cuenta la metodología de Práctica Clínica Basada en la Evidencia. la cual establece cinco pasos: 1. Establecimiento de una pregunta clínica, 2. Búsqueda de la mejor evidencia científica, 3. Análisis crítico de la mejor evidencia encontrada, 4. Transferencia de la evidencia científica y 5. Evaluación de la transferencia de la evidencia científica.

\section{Pregunta clínica}

La pregunta de revisión es ¿Cuál es la efectividad en la participación educativa y cuidado físico por parte de enfermería al estudiantado en centros de enseñanza especial para prevenir complicaciones de salud y potenciar el desarrollo?, la cual se desglosa en los siguientes elementos: fenómeno de interés y población:

Población: profesionales de enfermería que laboran en centros de enseñanza especial 


\section{Revista Electrónica Enfermeria Actual en costa Rica}

Fenómeno de interés: Participación educativa y cuidado físico de enfermería a la población de centros de enseñanza especial

El tipo de pregunta se clasifica como una pregunta de "Sombrilla" que se utiliza cuando se quiere abordar una amplia gama de temas relacionados con un tema de interés - en este caso, la participación educativa y cuidado físico del profesional de enfermería en los centros de enseñanza especial- para destacar si la base de la evidencia alrededor del tema de interés planteado es consistente, contradictoria o si existen resultados discrepantes del tema relacionado en el que se utiliza todo tipo de estudio para responderla.

\section{Criterios de inclusión}

Los criterios de inclusión para recuperar evidencia son los siguientes:

Población: esta revisión breve considerará estudios que incluyan profesionales de enfermería que laboran en centros de enseñanza especial, independientemente de su sexo o edad.

Fenómeno de interés: se considerarán estudios referentes a la participación educativa y el cuidado físico que realiza enfermería a la población de los centros de enseñanza especial.

\section{Tipos de estudio}

Se considerarán los estudios publicados tanto experimentales como no experimentales. En cuanto a los diseños, incluyen ensayos clínicos, ensayos clínicos aleatorizados, series de tiempo; además, se tomó en cuenta estudios observacionales que incluyen cohortes prospectivas y retrospectivas, casos y controles y estudios descriptivos que se complementan con reportes de casos individuales, cuya publicación esté en idioma español y en inglés.

\section{Estrategia de búsqueda}

La estrategia de búsqueda tendrá como objetivo encontrar estudios publicados para la que se seguirá tres pasos: inicialmente, se llevará a cabo una búsqueda limitada en MedLine, Google Scholar, EBSCO y Cochrane Plus por afinidad con el título de la investigación, además de contemplar que el resumen de la investigación contenga los elementos de relevancia -claramente expuestos- en este apartado, tales como el objetivo de la investigación, la metodología, resultados y conclusiones principales. Luego, se realizará una segunda búsqueda utilizando para ello los descriptores planteados en esta revisión en las bases de datos Clinical key, EMBASE, Lilacs, Medline vía EBSCO, Ovid, Pubmed, Scielo, Science Direct y Scopus. En tercer lugar, se buscará estudios adicionales en la lista de referencias de todos los informes y artículos identificados. Se incluirá toda la información disponible entre el 2013 al 2017. 


\section{Revista Electrónica Enfermeria Actual en costa Rica}

\section{Descriptores de búsqueda}

Para la búsqueda de información se utilizarán los siguientes descriptores: enfermería AND educación especial AND enseñanza especial, cuidados de enfermería AND centros de educación especial, tanto en español como en inglés.

\section{Selección de los estudios}

Luego de la búsqueda de información, se recopilará y se cargará todas las citas identificadas en el software bibliográfico Mendeley y se eliminará los duplicados. Luego, se examinará los títulos y los resúmenes para evaluarlos según los criterios de inclusión para la revisión. Se recuperará en su totalidad aquellos estudios que cumplan con los criterios de inclusión y se importará sus detalles en una matriz de datos de Excel. El texto completo de los estudios seleccionados se recuperará y evaluará en detalle en relación con los criterios de inclusión. Se excluirán los estudios de texto completo que no cumplan con los criterios de inclusión y se proporcionará los motivos de la exclusión en un apéndice del informe de la revisión final.

Los resultados de la búsqueda se anotará en su totalidad en el informe final y se presentará en un diagrama de flujo PRISMA 2009 en su versión en español.

\section{Extracción de los datos}

Se extraerá los datos de los artículos incluidos en la revisión de forma manual. Los datos extraídos incluirán detalles específicos, por ejemplo, año, autor, título, tipo y métodos del estudio, participantes, tipo de intervención, resultado principal y secundario de importancia para la pregunta clínica planteada y los objetivos específicos.

Se contactará a los autores de los documentos para solicitar datos faltantes o adicionales cuando sea necesario.

\section{Evaluación de la calidad metodológica}

La evaluación de la calidad de la investigación tiene por objetivo identificar la información que no tiene suficiente validez, debido a problemas en el diseño como es la rigurosidad metodológica deficiente, sesgos, inadecuada respuesta a los objetivos planteados. La herramienta que se utilizará para valorar la calidad de la información es la plataforma web "Fichas de Lectura Crítica 2.0" (FLC 2.0), la cual dirige paso a paso el proceso de lectura crítica: se trata de una aplicación web que apoya el desarrollo de la investigación basada en la evidencia a partir de la evaluación de la calidad de los documentos de interés para lectura crítica.

\section{Síntesis de datos}

Los resultados de esta revisión breve serán sintetizados y presentados en forma de tabla y resumen narrativo de acuerdo con los objetivos específicos para su análisis posterior. 


\section{Conflicto de intereses}

La autora declara que no tiene conflictos de tipo personal, comercial, académico, político o financiero.

\section{Agradecimiento}

Se le agradece a la Dra. Ligia Patricia Rojas Valenciano por su valioso aporte en la transmisión de esta metodología de investigación y apoyo para aplicarla.

\section{REFERENCIAS BIBLIOGRÁFICAS}

1. Del Val E. La enfermera en la escuela y la salud escolar. [Tesis Grado]. Cantabria, España: Universidad de Cantabria; 2015.

2. Campos M. Los primeros pasos hacia la enseñanza especial en Costa Rica. Rev. Actualidades Investigativas en Edu. 2015; 15(1). Disponible en http://www.scielo.sa.cr/pdf/aie/v15n1/a05v15n1.pdf

3. CENAREC. Convención internacional de Naciones Unidas sobre los derechos de las personas con discapacidad. 5ta ed. Costa Rica: PROLITSA; 2017.

4. Arce L, Barrientos R, Castillo M, Varela V. Intervención de Enfermería Pediátrica en el Centro Nacional de Rehabilitación. [Tesis de Postgrado]. San José, Costa Rica: Universidad de Costa Rica; 2006.

5. CONARE. Quinto informe del estado de la educación: Situación actual de los centros de educación especial en Costa Rica.2014.Disponible en:

https://estadonacion.or.cr/files/biblioteca_virtual/educacion/005/Lady_Melendez_et_al_Situacion_actual_centros _de_educ_espec.pdf.

6. Campos M. El papel de la enfermera comunitaria en el fomento de escuelas saludables: Justificación e intervenciones. Rev. Iberoamericana de enfermería comunitaria. 2016; 9(2). Disponible en: http://www.enfermeria21.com/revistas/ridec/articulo/27130/.

7. Katz S, Hayout I. Impact of an educational programme on nursing for children with developmental disabilities. Rev. The British Journal of Development Disabilities. 2016; 48(1). Disponible en http://www.tandfonline.com/toc/yjdd19/48/94

8. Macintosh $\mathrm{C}$, Thomas $\mathrm{C}$, Maughan E. Introduction to the special issue: Increasing the collaboration between school nurses and school personnel. Rev. Psycology in the Sch . 2015; 52(7). Disponible en http://onlinelibrary.wiley.com/doi/10.1002/pits.21852/abstract 


\section{Revista Electrónica Enfermeria Actual en costa Rica}

9.Litt J, Glymour M, Hauser-Cram P, McCormic M. The effect on impact health and development program on special education use at school age. Rev. The journal of pediatrics. 2014; Disponible en http://www.sciencedirect.com/science/article/pii/S0022347614009317

10. OMS. El desarrollo del niño en la primera infancia y la discapacidad: Un documento de debate. 2013. Disponible en http://www.who.int/disabilities/publications/early_childhood_disability/es/

11. UNICEF. Estado Mundial de la infancia 2013: Niños y niñas con discapacidad. Disponible en: https://www.unicef.org/sowc2013/files/SPANISH_SOWC2013_Lo_res.pdf

12. Vargas Z. La investigación aplicada: Una forma de conocer las realidades con evidencia científica. Rev. Educación 2009; 33(1). Disponible en: https://revistas.ucr.ac.cr/index.php/educacion/article/view/538

13. Rojas-Valenciano L, Cubero-ALpízar C, Leiva-Díaz V. Investigación secundaria: utilización de la mejor evidencia en la práctica clínica. 3ra ed. Costa Rica: CIEBE-UCR; 2017

14. Colegio de Enfermeras de Costa Rica. Código de Ética y Moral profesional del Colegio de Enfermeras de Costa Rica. Costa Rica; 2008. p. 41 